\title{
Drawing as an Academic Dialogue Tool for Developing Digital Learning Designs in Higher Education
}

\author{
Heidi Hautopp and Mie Buhl \\ IT, learning and design lab, Aalborg University, Copenhagen, Denmark \\ hhau@hum.aau.dk \\ mib@hum.aau.dk
}

\begin{abstract}
This paper reports on how drawing as an academic dialogue tool was explored as a crucial actor for driving design processes among humanistic master's students targeting their digital learning designs for online and blended learning contexts. The paper builds on a previous study that investigated students' use of self-produced visualisations during the digital design process. Although the study did not deal with visualisation and students were not trained to draw, the participants made extensive but unacknowledged use of visualisations. In the present study, a new group of students from the same master's programme were taught how to draw as a central component of the design process in order to investigate how this might expand their use of visual facilitation and drawing techniques to drive collaborative processes, design decisions and theoretical reflections. As design practices enter new interdisciplinary domains, in this case digital learning design, the aim was to explore how humanistic students can act as digital designers by adapting different design approaches and visual methods in particular. Likewise, the study offers an investigation of how students perceive these ways of working in an academic context. The empirical data, including teaching observations, students' visual productions and interviews with 27 students from nine groups after completing the course, were drawn primarily from an explorative case study in which master's students developed digital learning designs to solve a problem framed by an external stakeholder. Students' ways of producing visualisations in the different phases of their design process were analysed in terms of four design genres (explorative, investigative, explanatory and persuasive). The sociomaterial analysis traced how drawings and drawing activities unfolded during collaborative group processes which supported the development of digital learning designs. The findings confirmed the potential of drawing as a means for developing ideas, collaborating in different design phases and presenting and discussing design ideas with peers, target groups and external stakeholders. Furthermore, the findings revealed that drawing activities became a significant pedagogical consideration in the students' digital learning design and data collection process, where students balanced the interplay between initial analogue drawings and digital prototyping, testing their design concepts with target groups. The findings also showed that students perceived drawing and visual facilitation as practical tools but lacked an academic terminology for articulating these processes. The study suggests a need for substantial change to fully acknowledge the potential of drawing as an academic dialogue tool on the level with academic reading and writing when developing digital artefacts.
\end{abstract}

Keywords: visual facilitation, drawing as an academic dialogue tool, collaboration, digital learning design, higher education

\section{Introduction}

Western culture has consistently privileged the spoken and written word as the highest form of intellectual practice while regarding visual representations as second-rate illustrations of ideas (Mirzoeff, 2000; Bowen and Evans, 2015). Nevertheless, all scientific disciplines employ visualisations, and each discipline is characterised by a visual culture (e.g. Pauwels, 2006). For designers and architects, the act of drawing or 'sketching' is a familiar element of the iterative process of developing design products (Goldschmidt, 2003). Sketches are used both to reflect on and discuss ideas in design groups and when presenting design ideas to others (e.g. Schön, 1983; Tversky and Suwa, 2009). Within product design, sketches are also used to address and discuss users' experiences with digital products (Buxton, 2007). As design practices enter new interdisciplinary domains, such as learning design and communication design, researchers address the need for students to adapt concrete design methods when developing ideas (Hansen and Dalsgaard, 2012; Ejsing-Duun and Skovbjerg, 2019). When it comes to supporting the actual processes with learning how to design digital artefacts or processes, scholars of the humanities have tended to focus on texts and oral reflections on digital means and overlook the importance of the connected analogue means. Visual facilitation is one example of how drawing and visual methods are used to support group processes in organisations (e.g. Sibbet, 2008). They are based on the 1970s concept of graphic facilitation formulated by a group of organisational consultants in California (Qvist-Sørensen and Baastrup, 2020) who were inspired by how designers and architects utilise visualisations and sketching to present their ideas to clients (Sibbet, 2008). In the same way, visual facilitation can be understood as a point of entry to new organisational domains for design practices. However, little empirical research has been conducted on the use of visual facilitation as a formal learning method (Hautopp and Ørngreen, 2018). 
Other scholars have argued for the use of drawing and visual methods in education. Art Professor Betty Edwards (2012/1979) argued for teaching in drawing as an important part of our educational systems. Her work has received recognition within many fields, and her point of departure is neuropsychology, especially relating the act of drawing to Roger W. Sperry's work (1968) on brain hemispheres. Thus, teaching in drawing is mainly based on an individual cognitive argumentation on how our brain perceives and produces visualisations. In this paper, our focus is turned to the more collaborative aspect of using drawing in higher education as a crucial activity in designing digital artefacts. Visual facilitation involves the structured use of pen and paper methods to "facilitate interaction in a group of people, using structured visual content. It is a systematic way of drawing together with others" (Qvist-Sørensen and Baastrup, 2020, p. 20, our italics). Thus, in the field of visual facilitation, it is explicitly emphasised that the act of drawing should be accompanied with collaborative discussion among participants. As the visual facilitation has its origins in design, the teaching approach described in this paper draws on various design theories (e.g. Goldschmidt, 2003; Olofsson and Sjölen, 2007) and digital product design (Buxton, 2007). Furthermore, we refer to literature addressing both the term 'visual facilitation' and 'graphic facilitation', but use the term 'visual facilitation' to capture a broader definition of the field.

Previous studies of the use of visual methods in higher education in other domains of knowledge and practice (Gelting, Friis and Bang, 2015; Hyams, 2020; Hautopp and Ejsing-Duun, 2020) have shown that students from the design, architect and art fields benefit from using sketching and visual facilitation as academic practices, as these methods are familiar to them. The present study offers an investigation of the potential of visual facilitation among humanities students who were not familiar with drawing practices compared to design and art students. The inventive process of analogue drawings does not require wider skills: not necessarily a larger vocabulary or unlimited graphic techniques (Goldschmidt, 2003). Rather, what is required is an ability to use the representational act to reason and discuss design ideas (Goldschmidt, 2003; Buxton, 2007). Thus, we consider analogue drawings as an appropriate threshold for humanistic students to begin working as designers in crossdisciplinary fields. However, we argue that an introduction to the visual methods is crucial for students to rediscover and consider working this way in academic contexts. The aim was to explore how teaching drawing and visual facilitation can empower humanities students as digital learning designers by adapting visual methods for group work.

The exploratory case study was conducted in the master's programme 'IT, Learning and Organisational Change' (hereafter ILOO) in the Faculty of Humanities at Aalborg University, Denmark. The ILOO master's programme addresses research, development and the implementation of digital learning designs in a range of organisational and educational settings. Thus, it can be digital learning designs targeting the contexts of e.g. e-learning, flipped classrooms, video conferencing and so forth. ILOO master's students typically have a bachelor degree in pedagogy, teaching or computer science. Thus, they are skilled within those areas, but are not specifically trained in using drawing in an academic context. The course chosen for the study, 'IT and Learning Design', teaches students the theories of digital learning and education (e.g. Beetham, 2013) as well as design theories and methods (e.g. Kolko, 2010). As important elements of the course, the students were taught sketching, drawing methods and visual facilitation techniques for use in the digital design processes. In his book "Teaching in a Digital Age", Bates (2019) argues that the most important part of both classroom- and online teaching is how we design the learning environment focusing on collaboration. In this exploratory case study, the students were tasked to take the role of learning designers developing digital learning designs targeting different collaborative learning environments for the contexts of e-learning and flipped classrooms. Inspired by Bates' (2019) suggestions for experimenting with new digital opportunities, the students were encouraged to incorporate a range of different media in their digital learning designs such as text, graphics, audio, video and animation. However, as new technologies are developed and incorporated into media systems, old formats and approaches are carried over from older to newer media (Bates, 2019, p. 205). Thus, we investigated how analogue drawing formats can lay a basic foundation for the students to work visually in new digital formats when developing digital learning designs.

\section{Research design}

The exploratory case study was built on a Design Based Research approach (hereafter DBR) which focusing on both understanding and developing learning contexts. DBR is based on iterative pragmatic perspectives where researchers design and redesign pedagogical interventions, testing these in natural teaching settings (Brown, 1992). The iterative design of these interventions is a key feature of the knowledge production and results of a 
research project (Anderson and Shattuck, 2012; Barab and Squire, 2004). Thus, in the following the iterative design of interventions in this exploratory case study is described.

The exploratory case study was built on a previous study with other students in the same ILOO Course (Buhl, 2018), which did not teach visual facilitation and drawing techniques. The previous study showed that the students performed both analogue and digital visual practices during their design processes, but after finishing the course, they struggled to recall their use of visualisation when developing ideas, design drafts and prototypes. For instance, they had difficulty explaining their actions between the emergence and selection of design ideas and were able to recall the actual practices documented in their report only when the interviewer persisted in requesting examples. The diversity of visualisations identified in the study supports earlier evidence from elementary school settings (Meyer, 2016) showing how digital media support new visual practices, prompting new uses and representations of existing materialities. That study exposed the limitations of students' ability to articulate and reflect on their own visualisation practices, which were nevertheless identified as learning resources at all phases of the design projects.

In the present study, the same master's course was selected, as the aim was to explore how teaching visual facilitation and sketching might enhance students' collaborative processes by directing students' attention to the material aspects of drawing practice as a driver for ideation, collaboration, design and prototyping. Thus, an explicit focus was on drawing practices to promote visualisations as a more active participant in the students' meaning-making processes. To study the impact of teaching visual facilitation and sketching, a redesign was made of the course (Barab and Squire, 2004): Two drawing workshops were added to the course where the students and the teacher practiced drawing exercises together. Participatory observations were conducted during the workshops together with photo documentation of the students' and the teacher's drawing processes (Cresswell, 2011). After finalising the course, group interviews were conducted to generate knowledge about the students' drawing experiences. From following the activities in the drawing workshops, it was possible to explore the implications of providing an intensive introduction to drawing as a pedagogical intervention (Brown, 1992) to extend existing oral, writing and digital practices within the humanities. Furthermore, we could investigate how students experienced this intervention and how they reflected on the impact from the intervention after having finalised the course.

As we worked iteratively with researching pedagogical interventions, the investigative process involved producing demonstrable design and changes at the local level and reflecting on the use in other contexts (Barab \& Squire, 2004). Thus, the research approach is justified by the way the interventions worked in practice by providing a rich description of context, theory and interventions. Researchers within DBR argue that the rich documentation of interventions provides the readers of the research with a foundation to judge for themselves the possibility of achieving similar-or even better results-from the use of the interventions in their own contexts (Anderson and Shattuck, 2012, p. 17). In the analysis, we aim to give a rich and visual description of empirical examples discussed in relation to theories. Thus, the analysis address practice based consequences of the students' use of visual methods when developing digital learning designs for other educational researchers to judge the use of drawing interventions in their own contexts.

In the next section, the pedagogical intervention revolving the two drawing workshop is presented to give a rich introduction to the teaching context (Barab and Squire, 2004) as a point of departure for analyzing the students' reflective use of visual methods when developing digital learning designs.

\subsection{Pedagogical intervention: Drawing workshops}

The intervention was implemented during the 8-week course 'IT and Learning Design', in which groups of master's students were tasked to develop a digital learning design based on cases provided by external stakeholders. The work was organised as a design-based research process (e.g. Barab and Squire, 2004), involving iterations that included context research, design development, digital experiments and interventions, as well as theoretical reflection and documentation. 


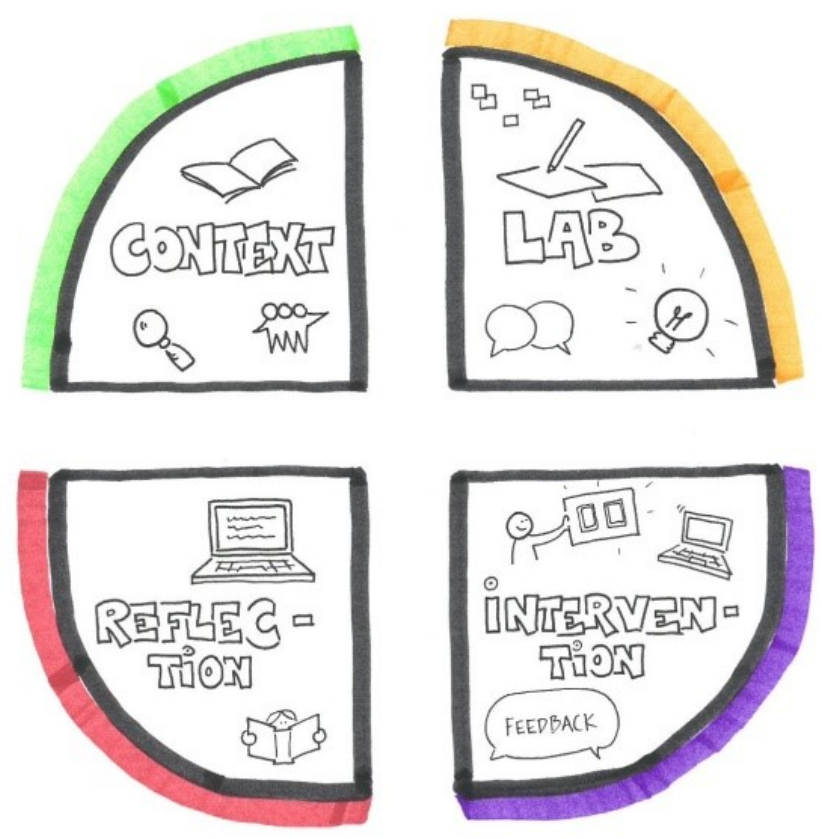

Figure 1: Visual presentation of the four phases in Design-based research (Hautopp and Buhl, 2020)

Thus, the course structure emphasised practice and theoretical knowledge generation as intertwined activities based on the logic of a design process (Buhl, 2016). Seventy students were enrolled in the course at the University's Aalborg and Copenhagen campuses. The drawing exercises were recorded by a document camera and live-projected to a wide screen and through video-conferencing systems at both campuses. To equip the students with tools for the different phases of the design-based research process, the intervention included two workshops introducing the students to visual facilitation through drawing exercises, design theories and feedback sessions. The first workshop focused on initial idea generation (Schön, 1983; Goldschmidt, 2003; Tversky and Suwa, 2009), while the second workshop focused more on the presentation of design ideas (QvistSørensen and Baastrup, 2020). The two workshop designs were based on Olofsson and Sjölen's (2007) mapping of four distinct design genres: investigative, explorative, explanatory and persuasive. These describe different modes of entry to the design process. The investigative and explorative genres are used to examine the design problem and to share design solutions within the design team. The explanatory genre is used to present and communicate a design concept to stakeholders outside the design team, and the persuasive genre relates to selling the concept in a marketing context (Vistisen, 2016). In the present study, the teaching intervention prompted students to use drawing in the different genres to gain hands-on experience as active participants by pragmatically testing and reflecting on the potentials and challenges of using visual methods in the design process.

Workshop 1: investigative and exploratory. At the outset, the phrase 'From head to paper-no need for fancy art' was used to emphasise that sketching and visual facilitation are about the act of developing, reflecting and communicating ideas rather than artistry (Valenzia and Adkins, 2009). As design proceeds from pragmatic ways of working (Hansen and Dalsgaard, 2012), the teacher first introduced drawing exercises involving the use of simple icons and elements to illustrate people, places and processes, using speech, text, colours and effects to highlight key words and elements (Qvist-Sørensen and Baastrup, 2020). 


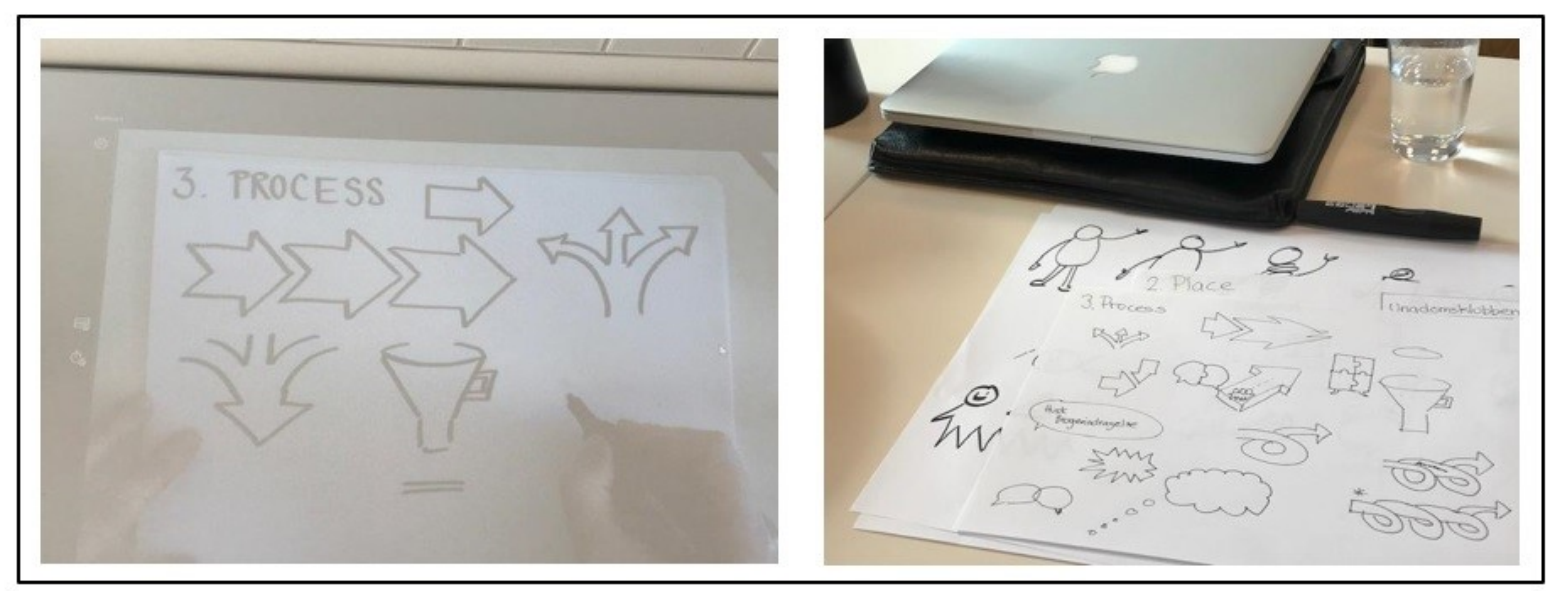

Figure 2: Wide-screen projection of teachers' hand drawings by document camera (left) and students' drawings (right)

Afterwards, the students were asked to investigate the design problem through drawings - first as a 10-minute individual assignment and then as a collaborative explorative exercise, in which they shared their drawings with each other and discussed ideas, potentials and barriers.

Workshop 2: explanatory and persuasive. As a point of departure, this workshop elaborated different theoretical perspectives on the use of drawings in educational settings, beginning with an introduction to visual facilitation (e.g. Sibbet, 2008) and how drawings and visual methods can be used for knowledge construction and representation (Bowen and Evans, 2015) in university teaching (Bang, Friis and Gelting, 2015). These theoretical concepts were combined with examples from the teacher's own empirical research and teaching in design and the humanities to show how drawings can be used as data collection tools for field notes (Causey, 2016) and interviews to elicit informants' visual imagery (Qvist-Sørensen and Baastrup, 2020). In the present study, the students were guided to use drawings in the different phases of their projects. After the presentation, they were prompted to draw more specific visuals related to aspects of their design problem (e.g. collaboration, digital devices, learning barriers and innovative and creative processes). In both workshops, the students were encouraged to offer feedback and to reflect on their hands-on drawing activities, relating these to the theories and methods of applying visual techniques in academic practices.

\subsection{The teacher as a role model for 'actually' drawing}

As our educational system has typically declined to use drawings as an acknowledged medium of learning after elementary school (Lyon, 2020), students in higher education may struggle to use drawings as they have unclear expectations of what is perceived as 'good', 'bad', or 'artistic' drawings, for example. Likewise, students may perceive drawings as something 'childlike' or 'childish' (Lyon, 2020, p. 5) due to the socio-cultural environment where they only associate the act of drawing with their childhood and elementary school. Opposite to students' experiences, we wanted to introduce drawing as a collaborative practice and as a crucial actor for designing digital artefacts. In this perspective, the teacher had an important task of being a 'role model' drawing together with the students not focusing on 'artistic' drawing, but instead directing drawings to the academic purposes of problem solving and collaboration (cf. the teacher's introduction phrase 'From head to paper - no need for fancy art', which was repeated throughout the course to minimize artistic expectations from the students). Frank and Madsen (2020) also expressed how the teacher's introduction to basic skills within visual facilitation slows down the pace and shows the process of drawing for the students to follow. In this paper, the teacher's use of a document camera and video conferencing made it possible for students across the two campuses to follow the drawing process while making their own basic drawings (see figure 2) as inspiration for implementing drawings in their further design processes. Thus, the workshops were not intended to deliver exact instructions for working with drawings, but to introduce a visual repertoire to develop students' skills and awareness regarding the use of visual methods as tools for design and collaboration in higher education. A further aim was to assess whether and how these drawing workshops would impact students' design activities during the rest of the course and to study drawing as an integrated part of digital designs. 


\subsection{From analogue drawings to digital prototypes}

Buxton's (2007) book about sketching user experiences revolve around product design with an emphasis on products that have a dynamic behaviour due to the incorporation of embedded digital technology. Buxton argued for a more experience-oriented design approach instead of an object-oriented one. In the context of this exploratory case study, students were tasked to develop digital designs to spur and support learning experiences among a target group.

Despite the accessibility of analogue drawing techniques (Goldschmidt, 2003) it is emphasized that the act of drawing sketches requires practice (Buxton, 2007), but teachers' introduction of basic drawing techniques is seldom prioritized in education (Edwards, 2012; Lyon, 2020). In this paper, we focus on introducing nondesigners - humanities students - to simple drawing techniques to give them a point of departure and inspiration to drawing their ideas in the initial design phases. The purpose of this 8-week course was not to educate them as full-blown designers, but to invite them to materializing their ideas in designerly ways (Hansen \& Dalsgaard, 2012); that is, producing, reading and redesigning through analogue drawings entangled with digital experiments.

Based on Buxton work (2007), we argue that sketches and prototypes are both instantiations of the students' digital learning design concepts. However, sketches dominate the early ideation phases, whereas prototypes are more concentrated at the later stages where things are converging within the design funnel (Buxton, 2007, p.139). As Olofsson and Sjölen (2007) expressed, design development represents the interplay between different genres where sketches and prototypes serve different purposes. In this exploratory case study, the focus was mainly on the students' development of the digital learning design concepts with less focus on the related attributes of cost, timelines, quantity and disposability and so on. which typically are tested through prototypes as more constituted designs (Buxton, 2007). Thus, the empirical data revolves primarily around the initial design phases as a crucial starting point for creating digital learning designs. Hence, this involved the students' reflection about the introduction of drawing techniques as a tool for the early design phases supporting the students' ideations and discussions of digital learning designs. Therefore, to work experience-oriented with different digital media, the students firstly were introduced to analogue drawing techniques (Qvist-Sørensen and Baastrup, 2020) to drive their initial ideation and discussion of different digital opportunities (Bates, 2019) before concretising their ideas in digital prototypes (Buxton, 2007).

After the two drawing workshops, the students were asked to work with different types of digital prototypes to further develop their digital learning designs based on their analogue drawings. Some of their choices included the Marvel app programme, the Sketch programme and Adobe XD. The connection between the analogue drawings as a foundation for developing digital prototypes will be elaborated on in the analysis. Buxton emphasised how prototypes, but also sketches, enable iterative user involvement, participation and testing much earlier in the design process (Buxton, 2007, p. 143). Thus, the analysis will contain examples of how students use different kinds of visual materializations of their digital learning design concepts when testing their ideas with peers and target groups.

\section{Theoretical framework}

In this section, the theoretical point of departure is outlined in order to analyse and discuss drawing as an academic dialogue tool and to address how this visual practice becomes a part of social learning processes.

\subsection{The social function of drawing in education}

To challenge academic privilege of the spoken and written word (cf. Mirzoeff, 2000; Bowen and Evans, 2015) we elaborate on why drawing should be acknowledged as an academic tool when students collaborate and acquire new knowledge in higher education. We seek to outline the potential and the research in drawing related to education and how insights should be expanded across disciplines.

In her PhD project "Learning through drawings - investigation into Danish Architecture Education" Inger Louise Berling Hyams (2020) investigated what role drawing plays in architectural education. Drawing has a special role in design and architecture education, since much of the learning and transfer of knowledge passes through drawing rather than regular language (Goldschmidt, 2003; Twersky and Suwa, 2009; Hyams, 2020). Thus, architectural educational discipline can be characterized as a field where the act of drawing is a commonly used practice and can serve as inspiration for other educational domains. 
As part of her PhD project, Hyams developed what she calls 'drawing epistemology' (Hyams, 2020, p. 196) which are linked to different historical paradigms of working within architectural education. Hyams explained that she puts 'drawing' first to emphasise that as an architect student, you achieve experiences through the act of drawing. Therefore, drawing is set before epistemology, rather than the more grammatically correct form of the 'epistemology of drawing', where epistemology comes first and not as a dependent of drawing. Here, Hyams also clarified the interrelation between the architect and the materials, defined by Donald Schön (1983) as the backtalk and dialogue with materials. Hyams' PhD project concentrated on architectural education and did not link the pedagogical considerations to other fields where particular drawing practices and the relation to thinking might be studied. However, our argument is that the concept of 'drawing epistemology' can be relevant to exploring within other disciplines in higher education.

Primarily, architectural drawings have an end goal of concrete buildings. Even though architects do not build buildings, they do drawings that are built for someone else (e.g. engineers and construction workers) to realise (Robbins, 1994, p.104). In this field, drawings have different functions ranging from abstract ideas to hard-line working drawings, which bridge different aspects of architectural practices (Robbins, 1994 in Hyams, 2020, p. 183). Based on Hyams' empirical work of interviewing architectural students, she concluded that students both see the drawings as an ongoing dialogue with their ideas (cf. Schön, 1983) and at the same time some students emphasise a purpose about that the drawing should be able to speak for themselves (Hyams, 2020, p. 184).

As we investigated the use of drawings when students worked with developing digital learning designs, the end goal was not merely concrete buildings (it might be an app or a website), but rather a suggestion for enhancing learning processes and collaboration processes. Thus, some kind of process is considered the end goal of the design process, where we consider visual facilitation as a relevant drawing genre for this purpose. Visual facilitation is not about depicting reality; instead, it is about representing ideas and icons in relation to other ideas illustrated on the basis of participants' contributions in the dialogue (Valenza and Adkins, 2009). Thus, the purpose of the drawings' self-communications is not as relevant, as drawings are always used and implemented in a dialogue surrounding the drawings (Qvist-Sørensen and Baastrup, 2020).

With their practice-based book Draw to Learn (2020), Nanna Frank and Anne Madsen made a teacher's guide for using graphic facilitation in educational settings. They take a point of departure in elementary school but wished to inspire anyone working with learning as a central part of their job. They emphasised the idea that in graphic facilitation, the use of drawings goes from being art or creative expression to becoming a strategic tool to enhance communication (Frank and Madsen, 2020). The visuals play an important role in the facilitation of complex contexts which are up for discussion with multiple stakeholders. Thus, the drawings are especially targeted to be a part of a dialogue centring on what the authors express as 'meaningful learning communities' (Frank and Madsen, chap. 1). The cases presented in the book are based on the authors' own experiences of using graphic facilitation, however, presented as fictional narratives. Thus, they present rich practice-based examples from elementary school, yet research is needed as well as examples from other educational contexts.

\subsection{Drawing as an academic dialogue tool}

In our study, broadening the educational contexts of where drawing practices are taught and being studied as a part of students' academic work was our aim - not least in the disciplines of digital design in the humanities that traditionally are oral and textual. Hyams' (2020) notion of 'drawing epistemology' was considered important in discussing the potential of drawing practices for learning. At the same time, when teaching of drawing was applied to the field of humanities, Frank and Madsen's (2020) notion of creating 'meaningful learning communities' became important to see drawing as an academic dialogue tool. Thus, we perceived visual facilitation as a systematic way of drawing together with others (Qvist-Sørensen and Baastrup, 2020) and as an academic driver (Hyams 2020) to enhance communication (Frank and Madsen, 2020) when students develop and present their digital learning design ideas (Olofsson and Sjölen, 2007; Buxton, 2007). As mentioned earlier, the basic drawing exercises were seen as an introduction to a visual repertoire to develop students' skills and awareness regarding the use of visual methods as an academic dialogue tool for design and collaboration in higher education. The social functions of the drawings are further elaborated upon in the next theoretical section. 


\subsection{Understanding drawing as social and material}

As addressed above, our approach to drawing was an academic dialogue tool characterized by being social, communicative and process-related. Understanding the students' visual actions was seen as situations of emerging meaning-making in the social and material interactions with the actual drawing and in dialogue with peers. Thus, visualisation was investigated as part of a the social learning process in which drawings and other materialities - including digital materialities - served as agents of meaning-making. Some scholars (e.g. Fenwick and Landri, 2012) have argued that we must look beyond the dichotomy between human intentionality and nonhuman objects for a fuller understanding of learning processes. Latour's (2005) actor-network-theory (ANT) prompted some researchers (e.g. Meyer, 2016) to adopt a broader perspective on empirical learning situations as complexes of social and material agency. Fenwick et al. (2011) argued that ANT offers the potential to rethink existing practices and to reframe conventional views of pedagogical practices in the humanities. They contended that learning consists of social material processes involving both human and non-human agency in a continuous flow of events (e.g. schedules, digital access codes, desks, pencils, stories, chewing gum and electricity) that participate in meaning-making. Rather than separate objects in a classroom, these were seen as collections of patterns of materiality that change and interact with human energies. Materialities, such as textbooks, tablets, discourses and learning models, offer different forms for participation in learning for a while, and these practices will in time spread to other learning environments and then later disappear. In this way, learning emerges from a continuous social practice of materialities of 'doing', to which meaning is attributed. Fenwick and Landri proposed the term hybrid assemblages to describe 'the continuum of materials, ideas, symbols, desires, bodies, natural forces, etc. that are always active, always reconstituting themselves' $(2012$, p. 3). In the present study, these hybrid assemblages served as the context for the empirical analysis of visualisations and their role in the design processes, tracing how drawings interact as active participants in the process. From this perspective, teaching, drawings, digital prototyping and the students' acts of drawing are entangled in the hybrid assemblage that constitutes the design process, including reservations towards the status of drawing in academia and the humanities (e.g. Mirzoeff, 2000; Bowen and Evans, 2015) and may be ideas of a requested aesthetic and selfcommunicative product (e.g. Hyams 2020). Furthermore, the hybrid assemblage may contain ideas of a childish preschool activity (Lyon, 2020). Therefore, investigating drawing as an academic dialogue tool in group processes requires a theory that captures the social dimension of drawing for learning. Our framework incorporated Wenger's (2000) account of social learning, which emphasises that learning happens socially and is negotiated through collaborative processes. This approach enabled us to explore how the students in our study attributed meaning to drawings and the act of drawing in combination with their digital design experiences when we interviewed them after completing the course.

\section{Analytical approach and empirical data}

Our suggestion of drawing as an academic dialogue tool for meaning-making was based on insights from practices within the professional domains of drawing. Together with sociomaterial and social learning theory, this constituted the framework for analysing the empirical data. Social material theory suggests overcoming the dichotomy between the analogue and digital material by approaching both as entangled actors in the hybrid assemblages. Before presenting our data analysis, it is useful to briefly outline our approach to the empirical material. Visual research is not only about the visual but also involves working through visuals and visualisations (Pauwels, 2006). For example, while semiotic analysis and content analysis are primarily used to explore visual objects themselves, field research is more appropriate for studying practices, experiences and processes related to the creation and utilization of those objects (Pauwels and Mannay, 2020). By tracing the visualisations as participants in hybrid assemblages, the sociomaterial perspective adopted here primarily focused on the students' practices and experiences rather than any thorough analysis of the visual artefacts themselves (e.g. composition and aesthetic qualities). The analysis concentrates on what Pauwels and Mannay (2020) described as production context and utilization context, tracing the becoming of the drawing and its different uses as an artefact in the four design genres (cf. Olofsson and Sjölen, 2007; Pauwels and Mannay, 2020; Fenwick and Landri, 2012).

The empirical data included participatory observations during drawing exercises, post-course group interviews with 27 students from nine groups of 2-4 students and the visual products of the group design processes. The interview guide was designed and aligned with the design based research approach (Barab and Squire, 2004) as the overall structure of the course, inviting the students to reflect upon their design processes and use of visual facilitation and sketching throughout the different design phases. The interview guide was planned by the authors on the basis of their participatory observations of teaching (Cresswell, 2011) and students' visual 
productions. However, to encourage students to speak more freely about their experiences, the interviews were conducted by a research assistant. The interviews were video-recorded and afterwards analysed by the authors, drawing on thematic analysis (Braun and Clarke, 2006). The following themes were identified in the empirical data which will be elaborated, analysed and discussed in the next sections:

- Drawing used as a driver in students' collaborative group work and theoretical reflections

- The drawings' diverse material agency for students' empirical data collection

- The role of analogue drawings and digital prototypes in students' feedback sessions

- Students' perception of drawing as a new mode of academic work

As part of the DBR approach, we have described the pedagogical intervention where drawing techniques and design theories where introduced to the students in two workshops (Barab and Squire, 2004). The interviews with students function as the primary focus for the empirical analysis on how the students experienced and reflected on the use of these visual methods for meaning making in their collaborative design processes.

\section{Analysis}

\subsection{Drawing used as a driver in students' collaborative group work and theoretical reflections}

Echoing previous findings (Buhl, 2018), students talked about how drawings concretised their ideas and functioned as participants in the negotiation of meaning (Wenger, 2000): 'It seemed like we were talking about the same thing, but when we visualised it, it turned out that we were not. It was the tool that helped us'. The drawings and the act of drawing also participated in group decision-making processes as the design elements became explicit: 'For each input, we talked about it and then sketched it. After it was sketched, one could see whether there was something wrong with it, or, well as soon as it was sketched, then one saw ... if it was okaydoes it work, or does it look confusing or stupid or something'. In this way, the students entered an exploratory dialogue with the materials (Goldschmidt, 2003; Olofsson and Sjölen, 2007), exemplifying the complex social and material agency of collaborative group processes (Meyer, 2016).

Another group emphasised the importance of drawing together: 'We all had the idea, the same idea. But when it came down on paper we realised it was not the same idea. Through the act of drawing together, we acquired a joint understanding, all three of us. It meant a lot for us'. Here, it can be argued how through the act of drawing together the students enhance their understanding and communication (Hyams, 2020; Frank and Madsen, 2020) of what seem to be a 'joint idea'. This group further elaborated on how the act of drawing became a significant actor in their idea development of a learning design app: 'Actually, in the beginning we only had ideas of the functions "spin" and "chair". We did not have the idea about "create", we only got that through drawing. Here, we became aware that something was missing (in the app)'. In this example, the drawings led to new ideas and became an important part in the social negotiation processes (Fenwick and Landri, 2012). Furthermore, the empirical data showed how the students were able to discuss, for example, the technical functionalities in their digital learning designs. A group emphasised how the act of drawing made it possible to discuss details about their digital learning design: 'The drawings created an overview and made it possible to discuss details (...) When you make drawings, it becomes evident how many elements and processes which are actually necessary when developing an app'. Another group explained how the quick drawings made the collaboration about the functions in the learning app more discussable: 'In the sketching phase, you can go into particularly details "Oh yes, we need a button there and what will be needed over here?"'. Here the student was imitating a group dialogue around the digital learning design, and it can be argued that the quick drawings made it possible to discuss the relation between the digital functionalities and possible user experiences (Buxton, 2007) in different utilization phases (Pauwels and Mannay, 2020). The students' experiences indicated an acceptance of drawing as a meaningful actor, and the interviews reported that it helped to maintain the students' focus in negotiating the multiple aspects regarding, for example, the relation between digital elements and users' learning experiences.

Maintaining a focus on their own drawing experiences enabled the students to reflect on the possibilities of integrating drawings as a modality in developing their learning designs. One group described how they integrated experiences from their own drawing processes into a digital learning design to facilitate teaching about innovation in an online learning context. Their external case related to teacher education; the design question asked how student teachers could be supported when developing teaching materials for innovation. Emphasising the material aspect of learning (Fenwick and Landri, 2012), one student said: 'You can talk about innovation, but how might you make a design about it? How can we make a product that supports [the process 
of innovation]? In other words, we can talk about it, but how should it look visually?'. The student explained how the group 'went through an innovative process' in using sketching and visual facilitation, and they later included this in their design of an innovation app for others to use as part of their own learning process. In other words, this group used their own production of visuals to reflect on their target group's utilization phase (Pauwels and Mannay, 2020). The example show how students reflected on and applied their own experiences of drawing in developing a learning design, and it can be argued that the drawings also prompted pedagogical considerations (Beetham, 2013) when the students took on the role of learning designers.

\subsection{The drawings' diverse material agency for students' empirical data collection}

In this section, we describe how drawing materialities were traced from group processes to participant involvement in data collection, with examples of how students used their own experiences of visual facilitation in the data collection phase. As mentioned earlier in the drawing workshop, the students were encouraged to use drawing activities both as part of their design processes and for empirical research (e.g. Qvist-Sørensen and Baastrup, 2020). The interviews further revealed that some of the groups used drawing activities in their initial field work, thereby involving their target group in the collaborative design process. One group of students described how they used various digital and analogue means to design prototypes in the exploratory and investigative modes (Olofsson and Sjölen, 2007) and later included drawing as a social activity in their field studies to involve participants in idea generation for the purposes of data collection: 'We had a user participant workshop in which participants made some sketches that we worked on in the Sketch programme later the same day. We included their sketches so that participants could see the process as well'.

During the process, the students moved between materialities, using hand drawings and the Sketch programme and switching between the four design genres (Olofsson and Sjölen, 2007), beginning with their own exploratory mode when preparing the workshop. Next, they invited participants to draw their ideas, leading later in the day to a more explanatory mode, where different ideas were presented and discussed using the Sketch programme.

From a sociomaterial perspective, the drawings' material agency can be seen as playing an important part in empirical knowledge creation. One student commented on the surprising insights they gained about the target group through the drawing exercises, as the drawing process opened a mutual space for reflection: 'I think that in every process shared with them, in every moment spent with them in a reflection space of some kind, there appeared some sort of groundbreaking new'. For instance, the group initially thought about including gamification elements in their digital learning design, but the participants' drawings and the subsequent discussion made it clear that the focus should be on accessibility and social activities.

One group commented on the new insights they gained into the elements of their design during a user workshop: 'We found that there were icons we made for the prototype that they could click around in while some of the icons we had used had to be replaced because they sent a different signal'. On that basis, the students adjusted the design to better suit the utilization context (Pauwels and Mannay, 2020). Another group reflected on how they integrated the participants' ideas in their final prototype: 'From the physical sketches they made, we talked a lot about how we could include their ideas. (...) The logo we made-actually, one of the young ones from the club made the logo that we chose to take further'. In this way, a participant's initial drawing achieved agency in the hybrid assemblage (Fenwick and Landri, 2012) that constituted the design concept targeted a blended learning context.

From a sociomaterial perspective, the drawings could be traced from the students' design processes to the participatory workshop, where the design ideas were developed and redesigned on the basis of participants' drawings and joint discussions. In this way, the drawings spread from one learning environment to another (Fenwick and Landri, 2012). In these processes, the student groups showed a flexible approach to the four design genres proposed by Olofsson and Sjölen (2007), with an increased focus on participants' experiences and feedback. This is also an example of how the students balanced the interplay between initial analogue drawings and digital prototyping in the Sketch programme, driving the design process from ideation to a more constituted design based on the participants' experiences (Buxton, 2007).

\subsection{The role of analogue drawings and digital prototypes in students' feedback sessions}

The above-mentioned examples show how students reflected on their own drawing experiences and how they related these to their development of digital learning designs and conducting data collection. Throughout the 
interviews, the students also elaborated on how the continuous peer feedback was an important driver for development in their design processes (e.g. feedback exercise from drawing workshop 2).

The visual materials were emphasised as an important actor of both driving the process and presenting ideas in the feedback sessions with other student groups and stakeholders, as the students in the different phases acquired new insights about their learning design (Fenwick and Landri, 2012; Wenger, 2000). One group explained how they went from one initial idea to another, which was largely reduced after a feedback session with fellow students and teachers. In that session, they went into explanatory mode (Olofsson and Sjölen, 2007) using their sketches as a dialogue tool (Frank and Madsen, 2020): 'My goodness, it was a large project. Our second prototype was huge and we could not explain it which we realised at the feedback seminar'. Due to the experience of not being able to explain their design, the students realised that they had to narrow their focus to create a digital learning design which should inspire a meaningful learning community (Frank and Madsen, 2020). Another group also reflected on receiving feedback on their design ideas: 'It was when we presented our design ideas to another student group that we realised that our design needs to be understandable for others. We know our idea, we know how the app works, or at least it works for us, so it was quite interesting to see what others did not immediately understand'. The feedback session enabled the students to discuss in more detail the utilization context of their digital learning design (Pauwels and Mannay, 2020), which supported the relation between the materialisation and the academic dialogue.

As the examples below will show, the students used different materialities when presenting their design ideas in the feedback sessions (e.g. analogue drawings as prototypes on paper, the Marvel app Programme, the Sketch Programme and Adobe XD). A group was observed showing how they produced and arranged different drawings as a prototype while presenting their work to the other student groups (opponent group) in a user test session. The user test showed that the drawings framed in a mobile telephone made the user experience explicit and easy for the test group to follow and comment on.

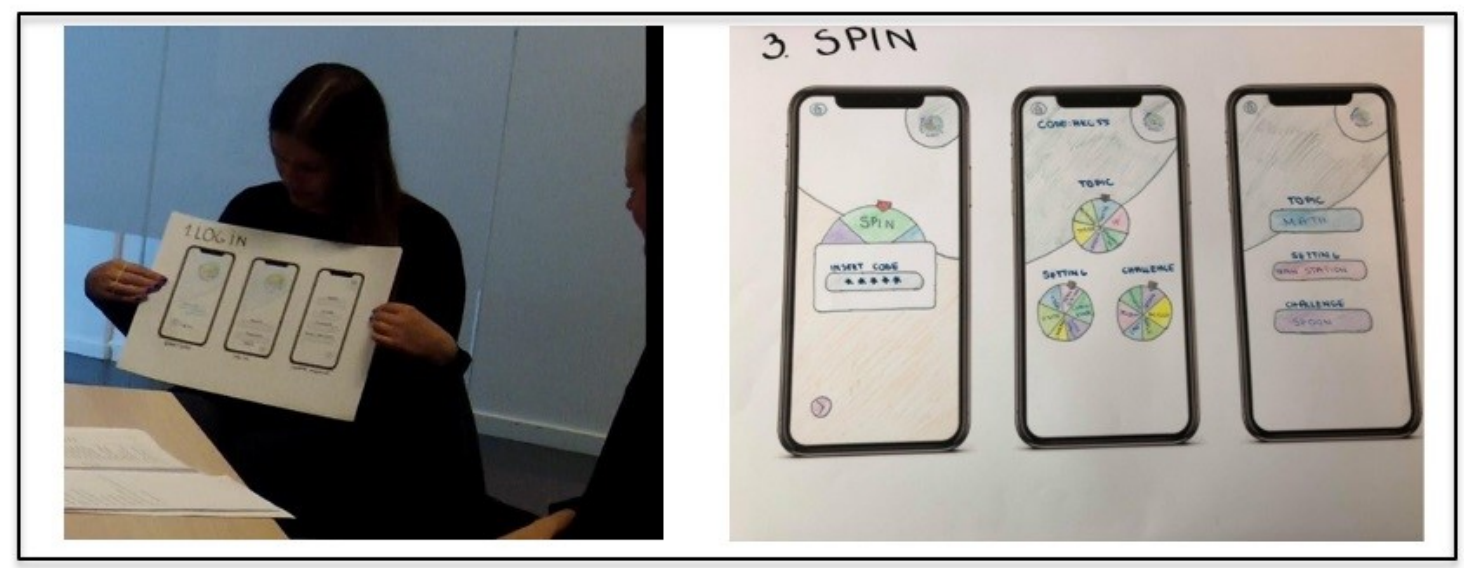

Figure 3: Students' prototype on paper showing a user experience of their digital learning design idea

The opponent group emphasised the following in their feedback: 'It was easy to follow your prototype on paper visualized as a mobile phone (...) I could easy follow from log-in to the different elements and activities in the app'. Another group had chosen to take the insights from their initial drawings phase and made it into a digital prototype in Adobe XD. Their opponent group emphasised the constituted format (Buxton, 2007) of the digital learning design: 'You have designed it very well; it looked like a real website and you explained it well. It was easy to follow your design ideas'. The opponent group addressed the fact that the students could press on the navigation buttons in the prototype in Adobe XD as "a dynamic experience". 


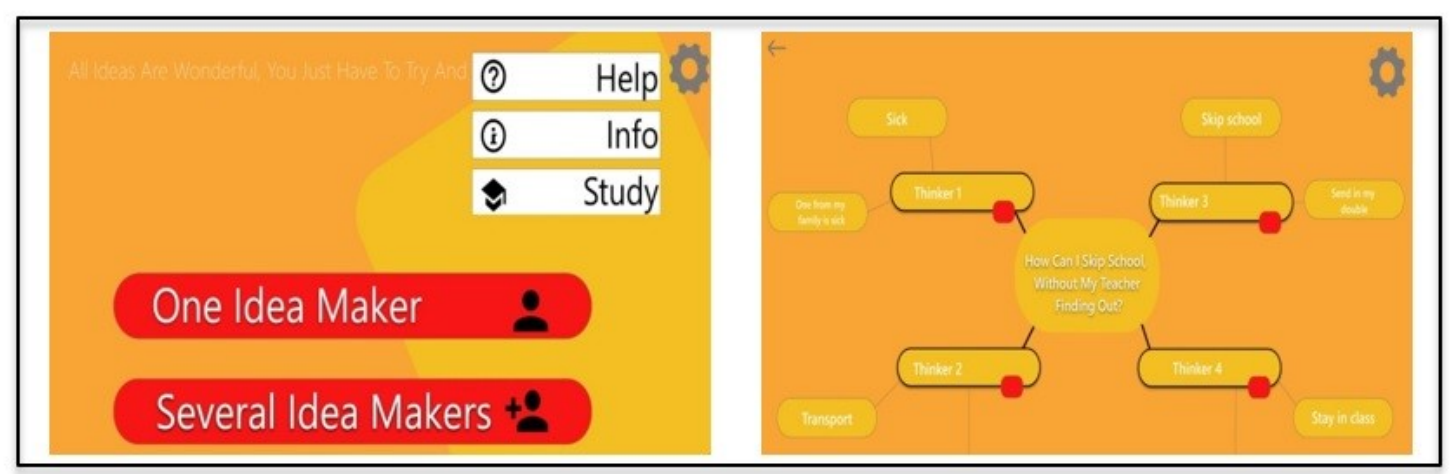

Figure 4: Students' prototype in Adobe XD showing a user experience of their digital learning design idea

The two groups had chosen differently when presenting their design ideas in the explanatory mode (Olofsson and Sjölen, 2007) and further discussed the different affordances of the modalities. Even though the dynamic character of the navigation in Adobe XD was appreciated, limitations were mentioned by the group who made the prototype, because 'you cannot do everything in a prototype programme'. Moreover, the analogue drawings in the prototype on paper was emphasised as crucial to snow the user experience: 'Your layout with the mobile phone made the user experience evident of what will follow in the next phase'. The student who had made the Adobe XD prototype explicitly expressed her reflection about the analogue format comparing it with the digital format: 'It think it works just as well as the digital format. I really liked that you have used drawings (...) I really enjoy drawing and I would like to become better'. The above-mentioned dialogue showed the students reflections about working from the analogue drawings in the initial design phases to a more constituted design format in the presentation drawings (Qvist-Sørensen and Baastrup, 2020) and the digital prototypes (Buxton, 2007).

Several groups expressed their appreciation of starting in the basic drawings, which led to further development and concretization of their digital learning design concepts. One group elaborated on how the feedback sessions showed the diversity in digital design solutions among the student groups: 'It was pretty amazing to see how different our design ideas had turned out' both in regard to the visual layout of the designs but also how the different student groups had solved the task framed by the external stakeholder. Another group elaborated: 'We have got a quite different perception of what the stakeholder needed compared to our opponent group. At the same time the designs have similarities because we still incorporated some of the same points (in the design). However, they are completely different; I find that enjoyable'. The examples showed how the drawings and visual representations became a crucial actor (Fenwick, 2012) when presenting a variety of design solutions. Likewise, the interviews showed the students' reflective experimentation of different analogue and digital media (Bates, 2019). Some students furthermore expressed a wish for more introductions to the different programmes for producing digital prototypes in the explanatory phase (Olofsson and Sjölen, 2007), which could guide a more constituted design presentations (Buxton, 2007). Thus, further studies could have a more specific focus on the production of digital prototypes based on the initial analogue drawings.

\subsection{Students' perception of drawing as a new mode of academic work}

The final analytical section will revolve around the students' perceptions on drawing as an academic dialogue tool for developing digital learning designs. Several students confirmed observations during the drawing workshop that the taught drawing exercises were meaningful and applicable to their own design processes, as in this example: 'We implemented several things from the teaching, so I think, that this...I think it made a lot of sense'. Others referred to how the drawings and visual facilitation guided their collaborative group processes: 'We realised that it was probably the lecture that was most beneficial...to guide us in the right direction and on the same path. It was simply an eye-opener as to how one could actually express one's ideas in another way'. From a sociomaterial perspective, the quotes generally confirmed how the entanglement of teaching exercises, drawings and discussions (Fenwick and Landri, 2012) became meaningful as the students reflected on how the act of drawing supported their collaborative design processes.

Several groups also addressed how the use of drawing was a new way for them to do academic work even though they did not approach the drawing practice theoretically. One group reflected on how they used drawings throughout the design processes and how this tool has been beneficial to learn: 'We have used 
sketching a lot throughout the design process and we think it is a really great tool we have learnt.' This group also emphasised how they used the drawing workshops as an important space for their design development and group discussions: 'Actually, we have used a part of the teaching to develop our design as we all did some sketching and then talked about our design'. Three other groups explicitly mentioned how the drawing workshops helped them overcome the barriers of drawing. One student explained, 'In general the two teaching workshops about sketching, it was really good. Because I think many of us had barriers like "fuck, I cannot draw" and "how do we ever get to sketch something?" and then she (teacher's name) showed drawings super simple; that was just really good".

As well as providing basic drawing skills and a visual repertoire, the findings indicate that the workshops lowered the students' barriers for drawing in an academic context. Thus, the students' reservations towards drawing (Lyon, 2020) were met by introducing simple drawing techniques (Qvist-Sørensen and Baastrup, 2020; Frank and Madsen, 2020) and by inviting the students to further develop their own visual repertoire suiting their design processes.

When asked directly about whether sketching theories had been a part of the students' design consideration, several groups rejected that it had been a part of their processes. They explained the more practical function of drawing in their group processes: 'getting ideas down on paper' and 'we probably used it more as a way to get clarity and insights about each other's understanding about the design ideas'. Even though the drawing exercises in the workshops were combined with theoretical and methodological theories within the field of design and visual facilitation (e.g. Goldschmidt, 2003; Twersky and Suwa, 2009; Qvist-Sørensen and Baastrup, 2020), the students' comments indicated that these combinations were not present in their experiences of the course.

The findings show that students reflected on how drawing as an academic dialogue tool for developing digital learning designs can be beneficial for their group learning processes and their design processes. They acknowledged their initial barriers towards the act of drawing and found the teaching valuable for engaging in the drawing practice. Despite the introduction of theory supporting the visual methods, the students mostly considered drawing, sketching and visual facilitation as practical methods.

\section{Discussion}

Our findings confirm that teaching visual facilitation and recall of students' drawing experiences helps students to realise the potentials of visualisations for learning as well as to explain their actions and selection of design ideas. The very activity of drawing has the potential to stage processes in which presuppositions can be tested, rejected and replaced by an open mind to address actual problems and serves as a prompt and direct way to share initial ideas and flows. Furthermore, the findings show that the drawing activity is entangled with digital activities as the students balanced the interplay between initial analogue drawings and digital prototyping e.g. in the Sketch programme. Concretisation in the different design phases promoted clarity in the development of ideas, facilitated collaborative processes and supported idea generation and discussion that were tested and further developed in combination with digital prototyping. Based on the initial analogue drawing techniques, a more thorough focus on developing digital prototypes (Bates, 2019) integrating digital sketching tools (Buxton, 2007 ) is suggested as a future research scope within the humanities.

When asked, the students lacked the theoretical and methodological terminology to specify what their drawing experiences achieved, as they used common language to narrate their actions. However, these narrations drew on the richness and diversity of visual materiality in driving social learning processes forward. For more theoretical reflections on the use of drawings and visual facilitation in academic practice, it can be argued that visual methods should be assigned a more prominent position as material participants on an equal footing with other materialities. This includes an extended knowledge about the theoretical foundation of drawing, which the students had not achieved. A continuous and explicit focus on relating practical drawing activities with theories are crucial to develop a more acknowledged visual learning culture in higher education.

By enlisting drawing as the primary materiality in this sociomaterial framework, this study can be understood as an instance of hybrid assemblage including both analogue and digital materialities. Nevertheless, this approach was found to be productive to the extent that it required us to focus on the actions occurring between the students and the drawings as a valuable encounter between human and non-human actors. In the student 
interviews, it was demonstrated how the drawing processes drove the design processes and took the collaborative work with the target group in new directions.

These findings also suggest that teaching drawing and visual facilitation as a pedagogical intervention impacts learning outcomes. Despite a lack of emphasis on visual education in Danish schools (Rasmussen, 2017), the students engaged with the workshop exercises and were able to reflect on them. Even though the students were positive towards the use of visual methods throughout their digital design processes, the findings also confirmed that these approaches were unfamiliar based on their previous educational experiences. If visual teaching activities are not continued as an integral part of their future courses, it remains to be seen to what extent students will continue to use drawing as an academic dialogue tool for learning, as the programme does not formally assess visual competences. Thus, curriculum organizers and teachers play an important part in maintaining a focus on drawing as an academic dialogue tool across academic disciplines.

Finally, the study showed that drawing activities became a significant pedagogical consideration, as students seemed more likely to use drawings as a tool for digital learning design and for involving their target group in the participatory workshops. Their flexible use of different design genres (Olofsson and Sjölen, 2007) indicates an interesting direction for further studies of what emerges when design practices enter new interdisciplinary domains (Hansen and Dalsgaard, 2012). As the empirical data in this study were limited, the intention was not to generalise the findings to other settings, but to investigate these students' understanding and use of visual methods in their digital design processes. As mentioned earlier, the results from this the exploratory case study can function as inspiration for applying similar interventions and visual methods to new local contexts in higher education (Anderson and Shattuck, 2012).

\section{Conclusion}

This paper provides empirical examples of how academic practices in higher education can benefit from a combination of different design methods, visual facilitation and drawing techniques as a means of enhancing students' development of digital learning designs. The study results are suggestive for teachers and researchers teaching design methods to students in educational contexts. From a sociomaterial perspective, the analysis followed students' drawings and the act of drawing through collaborative design processes and showed how these had implications for meaning- and decision-making when designing digital artefacts targeted different online- and blended learning contexts. The paper offers an investigation of drawing as an academic dialogue tool when developing digital learning designs, which was seen as playing an important part in students' pedagogical considerations about digital learning designs and data collection. Here, students balanced the interplay between initial analogue drawings and digital prototyping, testing their design concepts with peers and target groups. The present study confirms that visual facilitation has the potential to provide valued and valuable learning experiences, but further research is needed to assess the long-term implications of teaching drawing techniques and visual facilitation in such contexts. To support this research, future teaching in higher education would need a substantial change to fully acknowledge the potential of drawing as an academic dialogue tool on the level with academic reading and writing. Not least in the light of a growing digitalisation involving a range of different media in higher education. In this paper, we have shown the interplay between students' analogue drawing and their development of digital learning designs. Future research could expand the field of how analog practice may enhance digital practice in academic learning in the Humanities.

\section{References}

Anderson, T. and Shattuck, J. 2012, Design Based Research: A Decade of Progress in Educational Research? Educational Researcher, Vol. 41 (1) pp 16-25 DOI: 10.3102/0013189X11428813

Barab, S. and Squire, K. 2004. Design-based research: Putting a stake in the ground. Journal of the Learning Sciences, 13(1). p.1-14.

Bang, A.L., Gelting, A. K. G. and Friis, S. A. K., 2014. Designerly ways to theoretical insights: Visualisation as a means to explore, discuss and understand design theory. In: Design Research Society Conference. Umeå, Sweden, 2014. London: Design Research Society. Beetham, H. 2013. Designing for active learning in technology-rich contexts. In: H. Beetham and R. Sharpe, eds. Rethinking pedagogy for a digital age. Designing for 21st century learning (2nd ed.). New York: Routledge. pp 26-40.

Bates, T., 2019. Teaching in the digital age: Guidelines for designing teaching and learning. 2. Edition. Available through: https://pressbooks.bccampus.ca/teachinginadigitalagev2/ (Accessed: 29 June 2021)

Betty, E. 2012/1974. Drawing on the right side of the brain (4th ed.) New York: TarcherPerigee.

Bowen, T. and Ewans, M. 2015. What does knowledge look like? Drawing as a means of knowledge representation and knowledge construction. Education for Information, 31. pp 53-72. 
Braun, V. and Clarke, V. 2006. Using thematic analysis in psychology. Qualitative Research in Psychology, 3(2). pp.77101. DOI: $10.1191 / 1478088706$ qp063oa

Brown, A. 1992. Design experiments: Theoretical and methodological challenges in creating complex interventions in classroom settings. Journal of the Learning Sciences, 2 (2) pp 141-178.

Buhl, M. 2016. Theory-Generating Practice: Proposing a principle for learning design. Læring \& Medier, 15. pp 1-21.

Buhl, M. 2018. The role of visualizations for digital learning designs in collaborative group work. In I. A. Andreatos, C. Sgouropoulou and K. Ntalianis, eds. Proceedings of the 17th European conference on e-learning ECEL 2018. Reading, UK: Academic Conferences and Publishing International. pp.68-73.

Buxton, B. 2007. Sketching user experiences - getting the design right and the right design. Burlington, MA: Morgan Kaufmann, Elsevier.

Causey, A. 2016. Drawn to see: Drawing as an ethnographic method. Toronto: University of Toronto Press.

Cresswell, J. 2011. Educational research. planning, conducting, an evaluating quantitative and qualitative research (4th ed.). Boston, MA: Pearson Education.

Ejsing-Duun, S., \& Skovbjerg, H. M. 2019. Design as a mode of inquiry in design pedagogy and design thinking. The International Journal of Art \& Design Education, 38(2). pp.445-460. https://doi.org/10.1111/jade.12214

Fenwick, T., Edwards, R. and Sawchuk, P. 2011. Emerging approaches to educational research. Tracing the sociomaterial. New York: Routledge.

Fenwick, T. and Landri, P. 2012. Materialities, textures and pedagogies: Socio-material assemblages in education. Pedagogy, Culture \& Society, 20(1). pp.1-7.

Frank, N. and Madsen, A. 2020. Draw to learn. A guide for teachers and leaders who aspire to create curious and collaborative learning cultures using Graphic Facilitation. Copenhagen: Tools for Schools.

Goldschmidt, G. 2003. The backtalk of self-generated sketches. Design Issues, 19. pp.72-88.

Hansen, N.B. and Dalsgaard P. 2012. The Productive role of material design artefacts in participatory design events. In: Proceedings of the 7th Nordic Conference on Human-Computer Interaction: Making Sense Through Design (NordiCHI '12), ACM, New York. pp.665-674.

Hautopp, H. and Ørngreen, R. 2018. A review of graphic facilitation in organizational and educational contexts. Designs for Learning, 10(1). pp.53-62. https://doi.org/10.16993/dfl.97

Hautopp, H., \& Ejsing-Duun, S. 2020. Spaces of joint inquiry through visual facilitation and representations in higher education: An exploratory case study. Electronic Journal of E-Learning, 18(5). pp.373-386.

Hautopp, H. \& Buhl, M. 2020. Teaching visual facilitation and sketching for digital learning design in higher education. In: ECEL Conference. Available through: https://vbn.aau.dk/da/activities/presentation-of-paper-at-ecel-conferenceteaching-visual-facilita (Accessed 28 February 2021)

Hyams, I. L. B. 2020. Learning by drawing: Investigations into Danish architecture education. Roskilde: Roskilde Universitetsforlag.

Kolko, J. 2010. Abductive thinking and sensemaking: The drivers of design synthesis. Design Issues, 26(1). pp.15-28.

Latour, B. 2005. Reassembling the social: An introduction to actor-network theory. Oxford: Oxford University Press.

Lyon, P. 2020. Using drawing in visual research: Materializing the invisible. In: L. Pauwels and D. Mannay eds. The SAGE handbook of visual research methods. London: SAGE. pp.297-308.

Mirzoeff, N. 2000. An introduction to visual culture. New York: Routledge.

Meyer, B. T. 2016. Mobile devices and spatial enactments of learning: iPads in lower secondary schools. In I. I. Arnedillo and P. Isaias, eds. Proceedings of the 12th International Conference on Mobile Learning (1 udg., Bind 1, s. 3-10), Washington, DC: International Association for Development.

Olofsson, E. and Sjölen, K. 2007. Design sketching. Stockholm: KEEOS Design Books AB.

Rasmussen, H. 2017. Kompleks betydningsfremstilling i digitalt billedarbejde og billedæstetisk kompetenceudvikling i skolen [Complex meaning-making in digital visual production and visual aesthetic competence development] [Doctoral dissertation, Aalborg University]. Aalborg University Press.

Sibbet, D. 2008. Visual intelligence: Using the deep patterns of visual language to build cognitive skills. Theory Into Practice, 47. pp.118-127.

Tversky, B. and Suwa, M. 2009. Thinking with sketches. In: A. Markmann and K. Wood, eds. Tools for innovation. Oxford: Oxford Scholarship Online.

Pauwels, L. and Mannay, D. 2020. The Sage handbook of visual research methods. London: SAGE.

Pauwels, L. (Ed.) (2006) Visual cultures of science: Rethinking representational practices in knowledge building and science communication, Hanover NH/London: Dartmouth.

Qvist-Sørensen, O. and Baastrup, L. 2020. Visual collaboration. A powerful toolkit for improving meetings, projects, and processes. New Jersey: John Wiley \& Sons.

Robbins, E. 1994. Why architects draw. Cambridge, MA: Massachusetts Institute of Technology.

Schön, D. A. 1983. The reflective practitioner: How professionals think in action. London, UK: Temple.

Sperry, R.W. 1968. Hemisphere disconnection and unity in consciuos awareness. American Psychologist, 23. pp.723-733.

Valenza, C. and Adkins, J. 2009. Understanding visual thinking: The History and future of graphic facilitation. ACM Interactions, July and August. pp.39-45.

Vistisen, P. 2016. Sketching with animation. Using Animation to portray fictional realities aimed at becoming factual. Denmark: Aalborg University Press.

Wenger, E. 2000. Communities of practice: Learning, meaning, and identity. Cambridge: Cambridge University Press. 